TRANSACTIONS OF THE

AMERICAN MATHEMATICAL SOCIETY

Volume 353, Number 7, Pages 2725-2739

S 0002-9947(01)02693-9

Article electronically published on March 2, 2001

\title{
CENTRALIZERS OF IWAHORI-HECKE ALGEBRAS
}

\author{
ANDREW FRANCIS
}

\begin{abstract}
To date, integral bases for the centre of the Iwahori-Hecke algebra of a finite Coxeter group have relied on character theoretical results and the isomorphism between the Iwahori-Hecke algebra when semisimple and the group algebra of the finite Coxeter group. In this paper, we generalize the minimal basis approach of an earlier paper, to provide a way of describing and calculating elements of the minimal basis for the centre of an Iwahori-Hecke algebra which is entirely combinatorial in nature, and independent of both the above mentioned theories.

This opens the door to further generalization of the minimal basis approach to other cases. In particular, we show that generalizing it to centralizers of parabolic subalgebras requires only certain properties in the Coxeter group. We show here that these properties hold for groups of type $A$ and $B$, giving us the minimal basis theory for centralizers of any parabolic subalgebra in these types of Iwahori-Hecke algebra.
\end{abstract}

\section{IntRoduction}

The minimal basis for the centre of an Iwahori-Hecke algebra was defined and described in [F1], but a key part of the result required proving the existence of certain positive elements of the centre. The existence of these elements (the "class elements") was proved using the character theoretical results of [GR], and also used the existence of an isomorphism between the Iwahori-Hecke algebra over $\mathbb{Q}\left(q^{1 / 2}\right)$ and the group algebra $\mathbb{Q}\left(q^{1 / 2}\right) W$ (a result which needs some heavy machinery to prove).

In this paper, we give a new proof of the existence of the class elements, and using this prove the minimal basis results without need of characters, or even the isomorphism with the group algebra. Apart from the obvious advantage of avoiding the need for difficult theorems, this approach has at least one other advantage, that of generalizability. The methods used in this paper make the results of [F1] potentially generalizable to the centralizers of parabolic subalgebras, whereas the character theory approach is not. What is needed for a complete generalization of the minimal basis approach to centralizers is a generalization of the theorem by Geck and Pfeiffer GP on reducibility in conjugacy classes of Weyl groups. We prove in this paper that if $J$ is a subset of the set of simple reflections in type $A$

Received by the editors October 8, 1998 and, in revised form, December 21, 1999.

2000 Mathematics Subject Classification. Primary 20C33, $20 \mathrm{~F} 55$.

Key words and phrases. Coxeter groups, Iwahori-Hecke algebras, centre, centralizer, minimal basis.

The diagrams in this paper were created using Paul Taylor's Commutative Diagrams package. The research for this paper was in part supported by an Australian Postgraduate Award, and was done partially as part of work towards a Ph.D. at the University of New South Wales. 
and $B$, then all $J$-conjugacy classes are reducible, and this is sufficient to obtain the minimal basis theory for centralizers of the corresponding parabolic subalgebras in these cases.

After the general preliminaries of Section 1, we set about generalizing the Coxeter group result of Geck and Pfeiffer [GP to $J$-conjugacy classes in types $A$ and $B$.

The last two sections, 3 and 4, provide the minimal basis theory for centres of Iwahori-Hecke algebras in an entirely combinatorial way. Section 3 provides a proof of the existence of class elements using little more than linear algebra. This fact was previously only obtainable via character theory (see GR] and [F1]). Section 4 then finishes the proofs of the minimal basis theory, but avoids the use of the group algebra isomorphism, which was previously used (see [F1]). The only information about Iwahori-Hecke algebras needed for these two sections is the generators and relations, and the $[\mathrm{GP}]$ result, which makes these results entirely generalizable to all centralizers of parabolic subalgebras, pending an analogy to [GP]. Thus, these sections motivate the work on centralizers of parabolic subalgebras in Section 2.

I would like to thank Jie $\mathrm{Du}$, who encouraged me to look at this question while I was doing my Ph.D. under his supervision, and also Leonard Scott, who more recently gave his time to some discussions on these results, especially some of those in section 2. I would also like to thank Bob Howlett, who after reading narrower results in my thesis a long time ago suggested that it should be possible to generalize those results. Finally I'd like to thank the referee of the initial version of this paper, who suggested several good improvements.

Note: (2.5) and (2.6) have now been proved for $W$ any finite Coxeter group, thus similarly extending (4.6) to this generality (see [F2]).

\section{Preliminaries}

Let $W$ be a Weyl group with generating set $S$, and length function $l: W \rightarrow \mathbb{N}$. Then for $s, s^{\prime} \in S, W$ has the relations

$$
\begin{aligned}
s^{2} & =1, \\
\left(s s^{\prime}\right)^{m_{s s^{\prime}}} & =1,
\end{aligned}
$$

for some $m_{s s^{\prime}} \in \mathbb{N}$.

Each Coxeter group is partitioned into conjugacy classes $C$. For $J \subseteq S$, we may also partition $W$ into $J$-conjugacy classes, corresponding to sets of elements conjugate by elements of $W_{J}$. These are sometimes called orbits of $W_{J}$ under conjugation. We denote the set of all $J$-conjugacy classes in $W$ by $\operatorname{ccl}_{J}(W)$, and abbreviate $\operatorname{ccl}_{S}(W)$ to $\operatorname{ccl}(W)$. To distinguish reference to conjugacy classes and $J$-conjugacy classes, we will use $C$ to denote an $(S$ - $)$ conjugacy class, and $\mathfrak{C}$ for a general $J$-conjugacy class when $J$ may be a subset of $S$.

For $w \in W$, we write $\mathfrak{C}_{w}$ for the $J$-conjugacy class containing $w$. Let $l_{\mathfrak{C}}$ be the length of the shortest elements in the $J$-conjugacy class $\mathfrak{C}$, and let $\mathfrak{C}_{\min }$ be the set of shortest elements in $\mathfrak{C}$.

For any $J$-conjugacy class $\mathfrak{C}$ and $s \in J$ we can define an equivalence relation $\sim_{s}$ on $\mathfrak{C}$ by writing $w \sim_{s} u$ if $s w s=u$ and $l(w)=l(u)$. We then define the equivalence class $\sim_{J}$ to be generated by the relations $\sim_{s}$ for $s \in J$. The $\sim_{J}$-equivalence classes consist of elements of the same length which can be reached from each other by a sequence of conjugations by simple reflections from $J$, where each step in the sequence gives an element in $\mathfrak{C}$ of the same length. 
Each $J$-conjugacy class $\mathfrak{C}$ is the disjoint union of such $\sim_{J}$-equivalence classes, so we can specify the $\sim{ }_{J}$-equivalence class uniquely by choosing a representative from it. We will denote the $\sim{ }_{J}$-equivalence class containing $w$ by $\mathfrak{C}^{w}$.

For $w, w^{\prime} \in \mathfrak{C}$ for some $J$-conjugacy class $\mathfrak{C}$, we say $w \rightarrow_{J} w^{\prime}$ if there exist a sequence $r_{1}, r_{2}, \ldots, r_{m}$ of elements of $J$ and a sequence $w_{0}, \ldots, w_{m}$ of elements of $\mathfrak{C}$ such that if $w_{0}=w$, and $w_{i}=r_{i} w_{i-1} r_{i}(1 \leq i \leq m)$, then $w_{m}=w^{\prime}$, and $l\left(w_{i}\right) \leq l\left(w_{i-1}\right)$ with $w_{i} \neq w_{i-1}$, for $1 \leq i \leq m$.

(1.1) Definition. Let $\mathfrak{C}$ be a $J$-conjugacy class of $W$. We say $\mathfrak{C}$ is reducible if for all $w \in \mathfrak{C}$ there exists a $v \in \mathfrak{C}_{\text {min }}$ such that $w \rightarrow_{J} v$. Each $W_{J}-W_{J}$ double coset in $W$ is partitioned by $J$-conjugacy classes, and if every $J$-conjugacy class in the double coset $W_{J} d W_{J}$ is $J$-reducible, we say that the double coset $W_{J} d W_{J}$ is reducible.

(1.2) Theorem. Let $W$ be a finite Coxeter group.

(i) Every conjugacy class $C$ of $W$ is reducible.

(ii) If $w$ and $w^{\prime}$ are in $C_{\min }$, then there exist sequences of $x_{i} \in W$ and $w_{i} \in C_{\min }$ such that $w=w_{0}, x_{i} w_{i} x_{i}^{-1}=w_{i+1}$, and $w_{n}=w^{\prime}$, with either $l\left(x_{i} w_{i}\right)=l\left(x_{i}\right)+l\left(w_{i}\right)$ or $l\left(w_{i} x_{i}^{-1}\right)=l\left(w_{i}\right)+l\left(x_{i}^{-1}\right)$ for each $i$.

Proof. This was shown for all Weyl groups by Geck and Pfeiffer in GP, and for non-crystallographic types in GHLMP. The proof for classical types is by a general argument applied to each type, and the exceptional types are done using the GAP computer algebra package.

This theorem is generalized later in this paper to certain cases of $J$-conjugacy classes.

Let the set $\left\{\xi_{s} \mid s \in S\right\}$ be indeterminates, and let $R=\mathbb{Z}\left[\xi_{s}\right]_{s \in S}$. Then the Iwahori-Hecke algebra $\mathcal{H}$ over $R$ is the associative algebra generated by the set $\left\{\tilde{T}_{s} \mid s \in S\right\}$ with relations

$$
\tilde{T}_{s}^{2}=\tilde{T}_{1}+\xi_{s} \tilde{T}_{s}
$$

and

$$
\tilde{T}_{w}=\tilde{T}_{s_{i_{1}}} \cdots \tilde{T}_{s_{i_{r}}}
$$

when $w=s_{i_{1}} \cdots s_{i_{r}}$ is a reduced expression for $w$. We write $\mathcal{H}^{+}$for the set of elements of $\mathcal{H}$ whose terms $\tilde{T}_{w}$ have coefficients in $R^{+}:=\mathbb{N}\left[\xi_{s}\right]_{s \in S}$. That is, $\mathcal{H}^{+}$is the $R^{+}$-span of the set $\left\{\tilde{T}_{w} \mid w \in W\right\}$. Let $F:=\mathbb{Q}\left(\xi_{s}\right)_{s \in S}$.

[Note that $\mathcal{H}$ is a subalgebra of the Iwahori-Hecke algebra $\mathcal{H}_{q}$ over $\mathbb{Z}\left[q_{s}^{\frac{1}{2}}, q_{s}^{-\frac{1}{2}}\right]_{s \in S}$ obtained by setting $\xi_{s}=q_{s}^{\frac{1}{2}}-q_{s}^{-\frac{1}{2}}$ and $\tilde{T}_{s}=q_{s}^{-\frac{1}{2}} T_{s}$ for each generator $T_{s}$ of $\mathcal{H}_{q}$.]

There is a natural partial ordering on $\mathcal{H}^{+}$, induced by an ordering on elements of $R^{+}$, as follows. Let $\mathrm{Mon}_{j}$ be the set of all monomials in the $\xi_{s}$ for $s \in S$ of order $j$. If $a=\sum_{j, \mathfrak{m}_{j} \in \operatorname{Mon}_{j}} a_{\mathfrak{m}_{j}} \mathfrak{m}_{j}$ and $b=\sum_{j, \mathfrak{m}_{j} \in \operatorname{Mon}_{j}} b_{\mathfrak{m}_{j}} \mathfrak{m}_{j}$ for $a_{\mathfrak{m}_{j}}$ and $b_{\mathfrak{m}_{j}} \in \mathbb{N}$ are elements of $R^{+}$, then we say $a \leq b$ if and only if $a_{\mathfrak{m}_{j}} \leq b_{\mathfrak{m}_{j}}$ for each $j \in \mathbb{N}$ and $\mathfrak{m}_{j} \in \operatorname{Mon}_{j}$. Then if $h_{1}=\sum_{w \in W} a_{w} \tilde{T}_{w}$ and $h_{2}=\sum_{w \in W} b_{w} \tilde{T}_{w}$ with $a_{w}, b_{w} \in R^{+}$ are in $\mathcal{H}^{+}$, we say $h_{1} \leq h_{2}$ if and only if $a_{w} \leq b_{w}$ for all $w \in W$.

If $h \in \mathcal{H}^{+}$, we say $h$ is primitive if the monomial coefficients of terms $\tilde{T}_{w}$ in $h$ have no common factors. For example, $h=\xi_{s}^{2} \xi_{t} \tilde{T}_{w}+\xi_{s} \tilde{T}_{u}$ is not primitive, as the coefficients have a common factor of $\xi_{s}$. However, $\xi_{s} \xi_{t} \tilde{T}_{w}+\tilde{T}_{u}$ is primitive.

Given the partial order on the positive part of $\mathcal{H}$, the non-zero minimal elements under this partial order are well-defined, and we may similarly define the minimal 
elements of the positive part of any subalgebra of $\mathcal{H}$, for example, $Z(\mathcal{H})^{+}:=Z(\mathcal{H}) \cap$ $\mathcal{H}^{+}$. Define the set $Z(\mathcal{H})_{\min }^{+}$to be the primitive minimal elements of $Z(\mathcal{H})^{+}$. Then the main theorem of [F1] was that $Z(\mathcal{H})_{\text {min }}^{+}$is an $R$-basis for the centre of $\mathcal{H}$-and we called it the minimal basis.

Part of the structure introduced in [F1] to prove this result was a relatively simple basis for the centralizer of a generator $\tilde{T}_{s}$ in $\mathcal{H}$. For $d \in \mathfrak{D}_{\langle s\rangle,\langle s\rangle}$, the set of distinguished double coset representatives, define the following four types of element:

$$
\begin{aligned}
& \text { Type I, } d \in Z_{W}(s): \quad b_{d}^{I}=\tilde{T}_{d}, \\
& b_{d s}^{I}=\tilde{T}_{d s}, \\
& \text { Type II, } d \notin Z_{W}(s): \quad b_{d}^{I I}=\tilde{T}_{d}+\tilde{T}_{s d s}, \\
& b_{d s}^{I I}=\tilde{T}_{d s}+\tilde{T}_{s d}+\xi_{s} \tilde{T}_{s d s} .
\end{aligned}
$$

We call these the $s$-class elements because each specializes to a distinct $s$-conjugacy class.

A very useful result of [F1] which is a consequence of (1.2) is the following:

(1.3) Lemma. Suppose $w \in W$ is not minimal in its conjugacy class, and $h \in$ $Z(\mathcal{H})$. Then the coefficient of $\tilde{T}_{w}$ in $h$ is an $R^{+}$-linear combination of coefficients of strictly shorter elements in $h$. In fact, it is an $R^{+}$-linear combination of the coefficients of shortest elements of conjugacy classes.

We will use the following notation for the Weyl groups of types $A$ and $B$ :

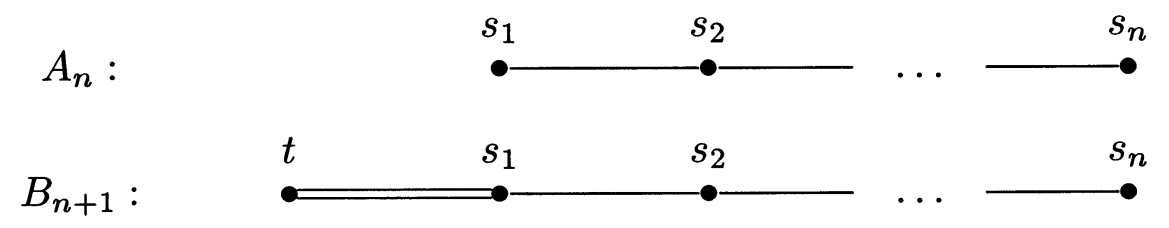

\section{Reducibility in $J$-COnJugacy Classes}

We will show in this section that for any subset $J$ of the set $S$ of simple reflections for a Weyl group of type $A$ or $B$ the $J$-conjugacy classes are reducible.

Each $J$-conjugacy class $\mathfrak{C}$ is contained in a unique $W_{J}-W_{J}$ double coset, so if $w \in \mathfrak{C}$ we may write $w=w_{1} d w_{2}$ with $d \in \mathfrak{D}_{W_{J}, W_{J}}, w_{i} \in W_{J}$, and $l(w)=l\left(w_{1}\right)+$ $l(d)+l\left(w_{2}\right)$. We can always reduce $w$ as far as $d w_{2} w_{1}^{-1}$ without increasing length, so it will suffice to ask the question, "Can we reduce an arbitrary $d u$ for $u \in W_{J}$ to a shortest element of its $J$-conjugacy class?".

Since $d$ is distinguished, the only way we can reduce length by conjugating $d u$ by some $s \in J$ is if $s d=d s^{\prime}$ for some $s^{\prime} \in J$, and if $s^{\prime} u s$ is shorter than $u$. This motivates us to consider reductions of the form $u \rightarrow s^{\prime} u s$ within $W_{J}$, and in general within a finite Coxeter group.

(2.1) Definition. Given a bijection $\sigma$ between subsets $J_{l}$ and $J_{r}$ of $S$, extended homomorphically to a map between $W_{J_{l}}$ and $W_{J_{r}}$, define the twisted $J_{l}$-conjugacy class corresponding to $\sigma$ and containing $w \in W$ to be

$$
\mathfrak{C}_{\sigma, w}:=\left\{\sigma(g) w g^{-1} \mid g \in W_{J_{l}}\right\}
$$


The operation previously mentioned sending $u$ to $s^{\prime} u s$ is the motivation for the above definition. It will transpire that reducibility in $J$-conjugacy classes reduces to reducibility in the above twisted conjugacy classes.

For $d$ distinguished in $W_{J} d W_{J}$, let $J_{l}:=\left\{s \in J \mid s d=d s^{\prime}, s^{\prime} \in J\right\}$, and let $J_{r}:=\left\{s^{\prime} \in J \mid s d=d s^{\prime}, s \in J_{l}\right\}$. Define the map $\sigma_{d}: J_{l} \rightarrow J_{r}$ by setting, for $s \in J_{l}, \sigma_{d}(s)=s^{\prime}$ when $s d=d s^{\prime}$. This $\sigma_{d}$ is clearly a bijection, and extends to a homomorphism naturally, since for $s_{1}, s_{2} \in J_{l}$ and $s_{i} d=d s_{i}^{\prime}$ we have $\left(s_{1} s_{2}\right) d=$ $s_{1} d s_{2}^{\prime}=d s_{1}^{\prime} s_{2}^{\prime}$, so that $\sigma_{d}\left(s_{1} s_{2}\right)=\sigma_{d}\left(s_{1}\right) \sigma_{d}\left(s_{2}\right)$.

(2.2) Lemma. Let $\sigma$ be any bijection $J_{l} \rightarrow J_{r}$ for subsets $J_{l}$ and $J_{r}$ of $J$, and let $u \in J_{l}$. Then $\sigma$ preserves length. That is, $l(\sigma(u))=l(u)$.

Proof. It suffices to consider $u \in J_{l}$ reduced in length. Since each simple reflection is mapped by $\sigma$ to a single reflection, we must have $l(\sigma(u)) \leq l(u)$. But since $\sigma$ is a bijection, we have an inverse map $\sigma^{-1}: J_{r} \rightarrow J_{l}$, and this also must satisfy $l\left(\sigma^{-1}(v) \leq l(v)\right.$ for $v \in J_{r}$. But then we have $l(u) \geq l(\sigma(u)) \geq l\left(\sigma^{-1}(\sigma(u))\right)=l(u)$, which proves the lemma.

The reason for an interest in such twistings is that when considering a $J$ conjugacy class contained in a double coset $W_{J} d W_{J}$, the conjugation by a simple reflection from $W_{J}$ can often be thought of as such a twisted conjugation on the group $W_{J}$. This relationship is shown in the following lemma. We show that a shortest element in a $J$-conjugacy class corresponds to a shortest element in a twisted $J_{l}$-conjugacy class.

In fact, our results are slightly more general than necessary. For the sake of induction, we actually show in this section that all twisted $J$-conjugacy classes are reducible in types $A$ and $B$. For the purposes of the rest of the paper, we only need the fact that the standard (un-twisted) $J$-conjugacy classes are reducible.

(2.3) Lemma. Let $d \in \mathfrak{D}_{J, J}$, and let $\sigma$ be a bijection from $J$ to $J^{\prime}$. Let $J_{l}^{\prime} \subseteq J^{\prime}$ be the set $\left\{s \in J^{\prime} \mid s d=d s^{\prime}\right.$ for some $\left.s^{\prime} \in S\right\}$, and let $\sigma_{d}$ be the bijection from $J_{l}^{\prime}$ to $J_{r}^{\prime}$ defined by $d$. Define the subset $J_{l}$ of $J$ to be $J_{l}=\sigma^{-1}\left(J_{l}^{\prime}\right)$.

Let $d w$ be an element of the twisted $J$-conjugacy class $\mathfrak{C}_{\sigma, d w}$, with $d \in \mathfrak{D}_{J, J}$ and $w \in W_{J}$. Then $d w$ is a shortest element of $\mathfrak{C}_{\sigma, d w}$ if and only if $w$ is a shortest element of its twisted $J_{l}$-conjugacy class $\mathfrak{C}_{\sigma_{d} \sigma, w}$.

Proof. Suppose $d w$ is shortest in $\mathfrak{C}_{\sigma, d w}$, but $w$ is not shortest in $\mathfrak{C}_{\sigma_{d} \sigma, w}$. Then there is an element $u$ of $W_{J_{l}}$ with the property that $l\left(\sigma_{d} \sigma(u) w u^{-1}\right)<l(w)$. But then $\sigma(u) d w u^{-1}=d \sigma_{d} \sigma(u) w u^{-1}$, since $u \in J_{l}$, and so

$$
\begin{aligned}
l\left(\sigma(u) d w u^{-1}\right) & =l\left(d \sigma_{d} \sigma(u) w u^{-1}\right) \\
& =l(d)+l\left(\sigma_{d} \sigma(u) w u^{-1}\right) \\
& <l(d)+l(w) \\
& =l(d w)
\end{aligned}
$$

which contradicts the assumption that $d w$ is shortest in its twisted $J$-conjugacy class.

On the other hand, suppose $w$ is shortest in its twisted $J_{l}$-conjugacy class, and let $u \in W_{J}$ be arbitrary. We may write $u=u_{1} u_{2}$ with $l(u)=l\left(u_{1}\right)+l\left(u_{2}\right)$, where 


$$
\begin{aligned}
& u_{2} \in W_{J_{l}} \text { and } u_{1} \in \mathfrak{D}_{W_{J} / W_{J_{l}}} \text {. Then } \sigma(u) d w u^{-1}=\sigma\left(u_{1}\right) d \sigma_{d} \sigma\left(u_{2}\right) w u_{2}^{-1} u_{1}^{-1}, \text { and so } \\
& \qquad \begin{aligned}
l\left(\sigma(u) d w u^{-1}\right) & =l\left(\sigma\left(u_{1}\right) d \sigma_{d} \sigma\left(u_{2}\right) w u_{2}^{-1} u_{1}^{-1}\right) \\
& =l\left(u_{1}\right)+l(d)+l\left(\sigma_{d} \sigma\left(u_{2}\right) w u_{2}^{-1} u_{1}^{-1}\right) \\
& \geq l\left(u_{1}\right)+l(d)+l\left(\sigma_{d} \sigma\left(u_{2}\right) w u_{2}^{-1}\right)-l\left(u_{1}^{-1}\right) \\
& =l(d)+l\left(\sigma_{d} \sigma\left(u_{2}\right) w u_{2}^{-1}\right) \\
& \geq l(d)+l(w) \\
& =l(d w),
\end{aligned}
\end{aligned}
$$

since $d$ is distinguished, and $w$ is minimal in its twisted $J_{l}$-conjugacy class. Thus $d w$ is minimal in its $J$-conjugacy class.

Remark. The proof above is a straightforward generalization of a proof given for a non-twisted case for principal parabolic subgroups in types $A$ and $B$ in [F2].

We now expand our attention to twisted conjugacy classes and twisted $J$ - conjugacy classes - the general case. After all, conjugacy classes and $J$-conjugacy classes are also twisted, but with a trivial twisting homomorphism. This shift to more generality makes some induction arguments easier.

(2.4) Lemma. Let $d \in \mathfrak{D}_{J, J}, J^{\prime}, J_{l}^{\prime}, J_{r}^{\prime}, J_{l}, \sigma: J \rightarrow J^{\prime}$, and $\sigma_{d}: J_{l}^{\prime} \rightarrow J_{r}^{\prime}$ be as in Lemma (2.3).

Then $d w$ is reducible in its twisted $J$-conjugacy class $\mathfrak{C}_{\sigma, d w}$ iff $w$ is reducible in its twisted $J_{l}$-conjugacy class $\mathfrak{C}_{\sigma_{d} \sigma, w}$.

Proof. If $w$ is reducible in its twisted $J_{l}$-conjugacy class $\mathfrak{C}_{\sigma_{d} \sigma, w}$, then there is a sequence of twisted conjugations by elements of $J_{l}, w \rightarrow \sigma_{d} \sigma(s) w s$, which reduces $w$ to a shortest element in $\mathfrak{C}_{\sigma_{d} \sigma, w}$ without increasing length.

It suffices to show that, for arbitrary $w \in W_{J}$, if $l\left(\sigma_{d} \sigma(s) w s\right) \leq l(w)$, then $l(\sigma(s) d w s) \leq l(d w)$. But $l(\sigma(s) d w s)=l\left(d \sigma_{d} \sigma(s) w s\right)=l(d)+l\left(\sigma_{d} \sigma(s) w s\right)$ since $d$ is distinguished, and this in turn is less than or equal to $l(d)+l(w)=l(d w)$. Consequently, every twisted conjugation by a simple reflection not increasing length in $\mathfrak{C}_{\sigma_{d} \sigma, w}$ provides a corresponding twisted conjugation by the same simple reflection, also not increasing length, in $\mathfrak{C}_{\sigma, d w}$.

The proof of this direction is completed by noting that since the sequence of twisted conjugations of $w$ ends with a shortest element of $\mathfrak{C}_{\sigma_{d} \sigma, w}$, the corresponding sequence in $\mathfrak{C}_{\sigma, d w}$ also ends in a shortest element by the above lemma. The reverse implication is similar.

(2.5) Theorem. Let $W$ be a finite Coxeter group of type $A$ or $B$, or a parabolic subgroup of a finite Coxeter group of type $A$ or $B$, let $S$ be the set of simple reflections generating $W$, and let $J \subseteq S$. Then every twisted $J$-conjugacy class in $W$ is reducible.

Proof. We start with the type $B_{n}$ case, and proceed by induction on $n$

The statement is trivially true for $B_{n}$ where $n=1$, and it is easy to check for $n=2$. So suppose it is true for all groups of type $B_{k}$ for $k<n$, and all parabolic subgroups of groups of type $B_{k}$. We need to show that every twisted $J$-conjugacy class is reducible in $W$ when $W$ is either $B_{n}$ or a parabolic subgroup of $B_{n}$.

Suppose initially that $J \varsubsetneqq S$, and let $\mathfrak{C}_{\sigma, u_{1} d u_{2}}$ be a twisted $J$-conjugacy class in $W$. Then $\mathfrak{C}_{\sigma, u_{1} d u_{2}} \subset W_{J^{\prime}} d W_{J}$ for some distinguished double coset representative $d$, and where $\sigma(J)=J^{\prime}$. 
For any $u_{1} d u_{2} \in \mathfrak{C}_{\sigma, u_{1} d u_{2}}$, we can reduce it to something of the form $d u$ as follows. Write $u_{1}=s_{i_{1}} \cdots s_{i_{r}}$ for $s_{i_{j}} \in J$. Twisted conjugation by $\sigma^{-1}\left(s_{i_{1}}\right)$ reduces the length of $u_{1}$ by one, and therefore either maintains the length of $u_{1} d u_{2}$ or reduces it by two. This can be repeated for $s_{i_{2}}$ up to $s_{i_{r}}$, leaving us with an element of form $d u$.

By (2.3), reducing an element of the form $d u$ in $\mathfrak{C}_{\sigma, u_{1} d u_{2}}$ is equivalent to reducing $u$ in its twisted $J_{l}$-conjugacy class $\mathfrak{C}_{\sigma_{d} \sigma, u}$ in $W_{J}$, where $J_{l}$ and $\sigma_{d}$ are as defined in (2.4). But since $J \varsubsetneqq S$, our induction hypothesis applies to the group $W_{J}$, and so the twisted $J_{l}$-conjugacy class is reducible in $W_{J}$.

It remains to check the case $J=S$. We need to show that all twisted conjugacy classes in $W=W\left(B_{n}\right)$ or a parabolic subgroup of $W$ are reducible. Let us first consider the case of a parabolic subgroup $W_{L}$ of $W$ (and we may assume $L \varsubsetneqq S$ ). This in fact reduces to cases already assumed by induction, as a conjugacy class of $W_{L}$ is actually an $L$-conjugacy class of $W$, sitting inside the trivial double coset $W_{L} 1 W_{L}$. So its reducibility is done since $L \varsubsetneqq S$.

Now consider the twisted conjugacy classes in $W=W\left(B_{n}\right)$. Here we are considering in fact twisted conjugacy classes $C_{\sigma}$, where $\sigma$ is a bijective homomorphism from $W$ to $W$-in other words, a group automorphism of $W$, or a graph automorphism of the Dynkin diagram of $W$. But in the case of type $B_{n}$, the only graph automorphism is the trivial one, and so the only twisted conjugacy classes are in fact the standard ones. In this case, with all the "twists" actually the identity, our twisted conjugacy classes are just the ordinary conjugacy classes, and these we know to be reducible by (1.2).

We are left to consider the type $A$ case. But every type $A$ Weyl group is a parabolic subgroup of a Weyl group of type $B$, and we have proved that all these and their parabolic subgroups have all twisted $J$-conjugacy classes reducible. Thus, the proof of the theorem is complete.

The following is a generalization of (1.2)(ii).

(2.6) Theorem. Let $W$ be of type $A$ or $B$, let $J \subseteq S$, and let $\sigma$ be a bijection from $J$ to $J^{\prime}$ which extends to an isomorphism of $W_{J}$ and $W_{J^{\prime}}$. If $w$ and $w^{\prime}$ are in $\mathfrak{C}_{\sigma, \min }$ for some twisted $J$-conjugacy class $\mathfrak{C}_{\sigma}$, then there exist sequences of $x_{i} \in W_{J}$ and $w_{i} \in \mathfrak{C}_{\sigma, \min }$ such that $w=w_{0}, \sigma\left(x_{i}\right) w_{i} x_{i}^{-1}=w_{i+1}$, and $w_{r+1}=w^{\prime}$, with either $l\left(\sigma\left(x_{i}\right) w_{i}\right)=l\left(x_{i}\right)+l\left(w_{i}\right)$ or $l\left(w_{i} x_{i}^{-1}\right)=l\left(w_{i}\right)+l\left(x_{i}^{-1}\right)$ for each $i, 0 \leq i \leq r$.

Proof. We proceed in a similar way to the previous theorem. It is easy to check for $W_{1}=W\left(A_{1}\right)$, or $W\left(B_{2}\right)$, so we may suppose inductively that it holds for all $W_{k}$ for $k<n$. We need to show that the theorem holds for $W_{n}$.

Let us first let $\mathfrak{C}_{\sigma}$ be a twisted $J$-conjugacy class, where $J \subset S$, and let $w=u d u^{\prime}$ be the reduced expression of any shortest element of $\mathfrak{C}_{\sigma}$, where $d$ is distinguished in $W_{J^{\prime}} w W_{J}$. Then $d u^{\prime} \sigma^{-1}\left(u^{-1}\right) \in \mathfrak{C}_{\min }$ also, and $l\left(d u^{\prime} \sigma^{-1}\left(u^{-1}\right)\right)=l\left(d u^{\prime}\right)+l(u)$. In other words, for any element $w=u d u^{\prime}$ of $\mathfrak{C}_{\min }$ there exists a $w^{\prime}=d v \in \mathfrak{C}_{\min }$ for which the claim is satisfied. This reduces the problem to showing that any pair $d v$ and $d v^{\prime}$ in $\mathfrak{C}_{\min }$ have the required property.

Now by (2.3), we have $d v \in \mathfrak{C}_{\sigma, \text { min }}$ if and only if $v$ is minimal in its twisted $J_{l}$-conjugacy class. Since $J_{l} \varsubsetneqq S$, we have the result by induction for $v$ and $v^{\prime}$ in $W_{J_{l}}$ and the sequence of conjugations by $x_{i}$ all in $W_{J_{l}}$. It follows that the result holds for $d v$ and $d v^{\prime}$.

If $J=S$, then this is a special case of (1.2)(ii), and the proof is complete. 
We have now shown a complete analogy of (1.2) in the case $W$ is of types $A$ or $B$. It seems quite likely that the result is true in general, that is, for all finite Coxeter groups. We hope to return to this question in later work.

\section{THE EXISTENCE OF CLASS ELEMENTS}

The remainder of this paper is devoted to Iwahori-Hecke algebras. First, in this section we will give a combinatorial account of the existence of the " $J$ class elements" - a result which is known in the case $J=S$ using the character theoretical results of GR.

In section 4 we will then use the existence of the $J$-class elements to prove the validity of the algorithm from [F1, and thus the minimal basis theory for all centralizers of parabolic subalgebras in types $A$ and $B$, including as a special case the central results from that paper. Previously in [F1] we used the isomorphism of $\mathcal{H}_{F}$ with $F W$ to obtain the dimension of the centre, but here we present the theory without this assumption.

We are going to prove results for $J$-conjugacy classes which satisfy certain properties - properties which are known to be true in the case $J=S$ (i.e., conjugacy classes) and in the case $W$ is of type $A$ or $B$ from the earlier parts of this paper. For convenience we will call them properties I and II, as they will appear verbatim in several different lemmas and theorems:

(3.0.1) Property I. All J-conjugacy classes are reducible.

(3.0.2) Property II. For any $J$-conjugacy class $\mathfrak{C}$ and $w, w^{\prime} \in \mathfrak{C}_{\min }$ there exist sequences of $x_{i} \in W_{J}$ and $w_{i} \in C_{\min }$ such that $w=w_{0}, w_{n}=w^{\prime}, x_{i} w_{i} x_{i}^{-1}=w_{i+1}$ and either $l\left(x_{i} w_{i}\right)=l\left(x_{i}\right)+l\left(w_{i}\right)$ or $l\left(w_{i} x_{i}^{-1}\right)=l\left(w_{i}\right)+l\left(x_{i}^{-1}\right)$ for all $1 \leq i \leq n-1$.

In the lemma below, (i) is an analogy of (1.3) (and is a direct consequence of Property I), and (ii) is a corollary of [F1, 3.2].

(3.1) Lemma. Suppose J satisfies Property I. Then:

(i) The coefficient of $\tilde{T}_{w}$ in an element $h$ of $Z_{\mathcal{H}}\left(\mathcal{H}_{J}\right)$ may be written as an $R^{+}$linear combination of coefficients of shortest elements of $J$-conjugacy classes in $h$.

(ii) Let $h \in Z_{\mathcal{H}}\left(\mathcal{H}_{J}\right)^{+}$, let $w \in \mathfrak{C}$, and let $a \in \mathbb{N}$. Then $a \tilde{T}_{w} \leq h$ implies $a \tilde{T}_{\mathfrak{C}} \leq h$.

The following is proved for centres in [J, (2.4)], using properties of Frobenius algebras, (and is almost certainly much older than that). A similar result is given in [DD], showing that certain norms are in the centralizer of the Iwahori-Hecke algebra in an $\mathcal{H}-\mathcal{H}$ bimodule. Here we give a combinatorial proof of the variation we need using the minimal basis for the centralizer of $\tilde{T}_{s}$ in $\mathcal{H}$.

(3.2) Lemma. Let $w \in \mathfrak{C}_{\min }$ for $\mathfrak{C} \in \operatorname{ccl}_{J}(W)$, with $J$ any subset of $S$. Then the element

$$
N_{W_{J}, 1}\left(\tilde{T}_{w}\right)=\sum_{u \in W_{J}} \tilde{T}_{u} \tilde{T}_{w} \tilde{T}_{u^{-1}}
$$

is in the centralizer $Z_{\mathcal{H}}\left(\mathcal{H}_{J}\right)$.

Proof. To show that $N=N_{W_{J}, 1}\left(\tilde{T}_{w}\right)$ is in the centralizer for $w \in \mathfrak{C}_{\min }$ for some $J$-conjugacy class $\mathfrak{C}$, we need to that show $N$ is in every centralizer $Z_{\mathcal{H}}\left(\tilde{T}_{s}\right)$ for $s \in J$. 
Consider the left cosets $\langle s\rangle d$ of $\langle s\rangle$ in $W_{J}$, with $s \in J$. Each left coset has exactly two elements, $d$ and $s d$, where $d$ is distinguished, and $W_{J}$ is partitioned by these left cosets. Let $\mathfrak{D}_{s}$ be the set of distinguished left coset representatives of $\langle s\rangle$ in $W_{J}$. Then we may write

$$
\begin{aligned}
N & =\sum_{u \in W_{J}} \tilde{T}_{u} \tilde{T}_{w} \tilde{T}_{u^{-1}} \\
& =\sum_{d \in \mathfrak{D}_{s}}\left(\tilde{T}_{d} \tilde{T}_{w} \tilde{T}_{d^{-1}}+\tilde{T}_{s d} \tilde{T}_{w} \tilde{T}_{d^{-1} s}\right) \\
& =\sum_{d \in \mathfrak{D}_{s}}\left(\tilde{T}_{d} \tilde{T}_{w} \tilde{T}_{d^{-1}}+\tilde{T}_{s}\left(\tilde{T}_{d} \tilde{T}_{w} \tilde{T}_{d^{-1}}\right) \tilde{T}_{s}\right) .
\end{aligned}
$$

Each product $\tilde{T}_{d} \tilde{T}_{w} \tilde{T}_{d^{-1}}$ is an $R$-linear combination of terms $\tilde{T}_{x}$ for $x \in W$, and so $N$ is an $R$-linear combination of terms of the form $\tilde{T}_{x}+\tilde{T}_{s} \tilde{T}_{x} \tilde{T}_{s}$, for $x \in W$. It now suffices to check that for every $x \in W$, the sum $\tilde{T}_{x}+\tilde{T}_{s} \tilde{T}_{x} \tilde{T}_{s}$ is in $Z_{\mathcal{H}}\left(\tilde{T}_{s}\right)$. This is an elementary task, and can be checked by going through the possibilities for $x$ in the double coset $\langle s\rangle x\langle s\rangle$, as follows.

Either $s x=x s$ or $s x \neq x s$. In the former case $x \in\left\{d^{\prime}, d^{\prime} s\right\}$ for $d^{\prime}$ distinguished in $\langle s\rangle x\langle s\rangle$, and in the latter $x \in\left\{d^{\prime}, d^{\prime} s, s d^{\prime}, s d^{\prime} s\right\}$.

If $s x=x s$ and $x=d^{\prime} \in \mathfrak{D}_{\langle s\rangle,\langle s\rangle}$, then

$$
\tilde{T}_{d^{\prime}}+\tilde{T}_{s} \tilde{T}_{d^{\prime}} \tilde{T}_{s}=\tilde{T}_{d^{\prime}}+\left(\tilde{T}_{d^{\prime}}+\xi_{s} \tilde{T}_{d^{\prime} s}\right)=2 b_{d^{\prime}}^{I}+\xi_{s} b_{d^{\prime} s}^{I} \in Z_{\mathcal{H}}\left(\tilde{T}_{s}\right) .
$$

If $s x=x s$ and $x=d^{\prime} s$ for $d^{\prime} \in \mathfrak{D}_{\langle s\rangle,\langle s\rangle}$, then

$$
\begin{aligned}
\tilde{T}_{d^{\prime} s}+\tilde{T}_{s} \tilde{T}_{d^{\prime} s} \tilde{T}_{s} & =\tilde{T}_{d^{\prime} s}+\tilde{T}_{s}\left(\tilde{T}_{d^{\prime}}+\xi_{s} \tilde{T}_{d^{\prime} s}\right) \\
& =2 \tilde{T}_{d^{\prime} s}+\xi_{s} \tilde{T}_{d^{\prime}}+\xi_{s}^{2} \tilde{T}_{d^{\prime} s}=\left(2+\xi_{s}^{2}\right) b_{d^{\prime}}^{I}+\xi_{s} b_{d^{\prime} s}^{I} \in Z_{\mathcal{H}}\left(\tilde{T}_{s}\right) .
\end{aligned}
$$

If $s x \neq x s$ and $x=d^{\prime} \in \mathfrak{D}_{\langle s\rangle,\langle s\rangle}$, then

$$
\tilde{T}_{d^{\prime}}+\tilde{T}_{s} \tilde{T}_{d^{\prime}} \tilde{T}_{s}=\tilde{T}_{d^{\prime}}+\tilde{T}_{s d^{\prime} s}=b_{d^{\prime}}^{I I} \in Z_{\mathcal{H}}\left(\tilde{T}_{s}\right) .
$$

If $s x \neq x s$, and $x=s d^{\prime}$ for $d^{\prime} \in \mathfrak{D}_{\langle s\rangle,\langle s\rangle}$, then

$$
\tilde{T}_{s d^{\prime}}+\tilde{T}_{s} \tilde{T}_{s d^{\prime}} \tilde{T}_{s}=\tilde{T}_{s d^{\prime}}+\tilde{T}_{d^{\prime} s}+\xi_{s} \tilde{T}_{s d^{\prime} s}=b_{d^{\prime} s}^{I I} \in Z_{\mathcal{H}}\left(\tilde{T}_{s}\right) .
$$

If $s x \neq x s$, and $x=d^{\prime} s$ for $d^{\prime} \in \mathfrak{D}_{\langle s\rangle,\langle s\rangle}$, then

$$
\tilde{T}_{d^{\prime} s}+\tilde{T}_{s} \tilde{T}_{d^{\prime} s} \tilde{T}_{s}=\tilde{T}_{d^{\prime} s}+\tilde{T}_{s d^{\prime}}+\xi_{s} \tilde{T}_{s d^{\prime} s}=b_{d^{\prime} s}^{I I} \in Z_{\mathcal{H}}\left(\tilde{T}_{s}\right) .
$$

If $s x \neq x s$, and $x=s d^{\prime} s$ for $d^{\prime} \in \mathfrak{D}_{\langle s\rangle,\langle s\rangle}$, then

$$
\begin{aligned}
\tilde{T}_{s d^{\prime} s}+\tilde{T}_{s} \tilde{T}_{s d^{\prime} s} \tilde{T}_{s} & =\tilde{T}_{s d^{\prime} s}+\tilde{T}_{s}\left(\tilde{T}_{s d^{\prime}}+\xi_{s} \tilde{T}_{s d^{\prime} s}\right) \\
& =\tilde{T}_{s d^{\prime} s}+\tilde{T}_{d^{\prime}}+\xi_{s} \tilde{T}_{s d^{\prime}}+\xi_{s} \tilde{T}_{d^{\prime} s}+\xi_{s}^{2} \tilde{T}_{s d^{\prime} s} \\
& =\left(\tilde{T}_{d^{\prime}}+\tilde{T}_{s d^{\prime} s}\right)+\xi_{s}\left(\tilde{T}_{s d^{\prime}}+\tilde{T}_{d^{\prime} s}+\xi_{s} \tilde{T}_{s d^{\prime} s}\right) \\
& =b_{d^{\prime}}^{I I}+\xi_{s} b_{d^{\prime} s}^{I I} \in Z_{\mathcal{H}}\left(\tilde{T}_{s}\right) .
\end{aligned}
$$

(3.3) Lemma. The set $\left\{N_{W_{J}, 1}\left(\tilde{T}_{w_{\mathfrak{C}}}\right) \mid \mathfrak{C} \in c c l_{J}(W), w_{\mathfrak{C}} \in \mathfrak{C}_{\min }\right\}$ is linearly independent over $F$, and is contained in $Z_{\mathcal{H}}\left(\mathcal{H}_{J}\right)$. 
Proof. The centrality follows from (3.2).

The only summands in the full expansion of $N_{W_{J}, 1}\left(\tilde{T}_{w_{\mathfrak{C}}}\right)$ with coefficient without a factor of $\xi_{s}$ for some $s \in S$ are those from $\mathfrak{C}$. Thus, on specializing to $\xi_{s}=0$ for each $s$, we have a sum of elements in the $J$-conjugacy class. The set of all sums of elements in $J$-conjugacy classes is linearly independent (it forms a basis for the centralizer of $F W_{J}$ in the group algebra $F W$ ), and so the norms are also linearly independent.

We want to find elements of $Z_{\mathcal{H}}\left(\mathcal{H}_{J}\right)$ which are analogous to $J$-conjugacy class sums in the group algebra. The following elements will turn out to fill that role.

(3.4) Definition. If $\Gamma_{\mathfrak{C}} \in Z_{\mathcal{H}}\left(\mathcal{H}_{J}\right)^{+}$, then $\Gamma_{\mathfrak{C}}$ is called a $J$-class element if it satisfies the following two properties:

(3.4.1) $\left.\Gamma_{\mathfrak{C}}\right|_{\xi_{s}=0, s \in S}=\tilde{T}_{\mathfrak{C}}$, and

(3.4.2) $\Gamma_{\mathfrak{C}}-\tilde{T}_{\mathfrak{C}}$ contains no terms of shortest length in any $J$-conjugacy class.

The purpose of this section is to prove such elements exist.

For some fixed $h=\sum_{w \in W_{J}} r_{w} \tilde{T}_{w} \in Z \mathcal{H}\left(\mathcal{H}_{J}\right)$, define the function $\mathfrak{h}: \mathcal{H} \rightarrow R$ by setting $\mathfrak{h}\left(\tilde{T}_{w}\right)=r_{w}$ and extending linearly to the whole of $\mathcal{H}$.

(3.5) Lemma. For all $w \in W$ and $v \in W_{J}, \mathfrak{h}\left(\tilde{T}_{w} \tilde{T}_{v}\right)=\mathfrak{h}\left(\tilde{T}_{v} \tilde{T}_{w}\right)$.

Proof. We first prove this for all $w \in W$ when $l(v)=1$, setting $v=s \in J$. It suffices to consider $w \in\langle s\rangle d\langle s\rangle$ for some $d \in \mathfrak{D}_{\langle s\rangle,\langle s\rangle}$. Suppose first that $d s=s d$. Then $w=d$ or $d s$, and in fact we have $\tilde{T}_{d} \tilde{T}_{s}=\tilde{T}_{s} \tilde{T}_{d}$ and $\tilde{T}_{d s} \tilde{T}_{s}=\tilde{T}_{s} \tilde{T}_{d s}$, so the lemma follows trivially. So we may suppose $d s \neq s d$, and thus $w=d, d s, s d$, or $s d s$. Then, using [F1 3.1], we have

$$
\begin{aligned}
\mathfrak{h}\left(\tilde{T}_{d} \tilde{T}_{s}\right) & =\mathfrak{h}\left(\tilde{T}_{d s}\right)=r_{d s}=r_{s d}=\mathfrak{h}\left(\tilde{T}_{s} \tilde{T}_{d}\right), \\
\mathfrak{h}\left(\tilde{T}_{d s} \tilde{T}_{s}\right) & =\mathfrak{h}\left(\tilde{T}_{d}+\xi_{s} \tilde{T}_{d s}\right)=\mathfrak{h}\left(\tilde{T}_{d}\right)+\xi_{s} \mathfrak{h}\left(\tilde{T}_{d s}\right)=r_{d}+\xi_{s} r_{d s}=r_{s d s}=\mathfrak{h}\left(\tilde{T}_{s} \tilde{T}_{d s}\right), \\
\mathfrak{h}\left(\tilde{T}_{s d s} \tilde{T}_{s}\right) & =\mathfrak{h}\left(\tilde{T}_{s d}+\xi_{s} \tilde{T}_{s d s}\right)=r_{s d}+\xi_{s} r_{s d s}=r_{d s}+\xi_{s} r_{s d s}=\mathfrak{h}\left(\tilde{T}_{s} \tilde{T}_{s d s}\right) .
\end{aligned}
$$

The case $w=s d$ is exactly symmetric to the $w=d s$ case above.

Now suppose the lemma holds for all $w \in W$ when $l(v) \leq k$, and suppose $x \in W_{J}$ has length $l(x)=k+1$. Then $x=v s$ for some $v$ of length $k$ and some $s \in J$; that is, $l(x)=l(v)+l(s)$, and $\tilde{T}_{x}=\tilde{T}_{v} \tilde{T}_{s}$. Let $w \in W$. Then $\mathfrak{h}\left(\tilde{T}_{w} \tilde{T}_{x}\right)=\mathfrak{h}\left(\tilde{T}_{w} \tilde{T}_{v} \tilde{T}_{s}\right)=$ $\mathfrak{h}\left(\tilde{T}_{s} \tilde{T}_{w} \tilde{T}_{v}\right)=\mathfrak{h}\left(\tilde{T}_{v} \tilde{T}_{s} \tilde{T}_{w}\right)=\mathfrak{h}\left(\tilde{T}_{x} \tilde{T}_{w}\right)$, with the second and third equalities following since $\tilde{T}_{w} \tilde{T}_{v}$ (resp. $\tilde{T}_{s} \tilde{T}_{w}$ ) are linear combinations of elements $\tilde{T}_{u} \in \mathcal{H}$, and $\mathfrak{h}$ is linear. So by induction we may pass $\tilde{T}_{s}$ and $\tilde{T}_{v}$ respectively through terms in the products $\tilde{T}_{w} \tilde{T}_{v}$ and $\tilde{T}_{s} \tilde{T}_{w}$ respectively. This proves the lemma.

It is well known that the terms corresponding to shortest elements of a conjugacy class in a central element have the same coefficient. This has been proved by Ram $[\mathrm{R}$ ] and Starkey (see [C]) in type $A$, and by Geck and Pfeiffer [GP for general Weyl groups. These results used the standard trace map $\tau$ defined by setting $\tau\left(\tilde{T}_{x}\right)=1$ if $x=1$ and 0 if $x \neq 1$, and it is possible to obtain the following result for the case $J=S$ using $\tau$. In fact, if we take $h=1$ for our central element $(J=S)$, then the corresponding $\mathfrak{h}$ is exactly the standard $\tau$.

(3.6) Lemma. Let $(W, S)$ be a finite Coxeter system, and let $J \subseteq S$ be such that all $J$-conjugacy classes satisfy Property II (3.0.2). Let $w, w^{\prime} \in \mathfrak{C}_{\min }$ for some $J$ conjugacy class $\mathfrak{C}$. Then, if $h=\sum_{w \in W} r_{w} \tilde{T}_{w} \in Z_{\mathcal{H}}\left(\mathcal{H}_{J}\right)$, we have $r_{w}=r_{w^{\prime}}$. 
Proof. By Property 2 of $J$-conjugacy classes, we have the existence of sequences of $x_{i} \in W_{J}$ and $w_{i} \in C_{\min }$ such that $w=w_{0}, w_{n}=w^{\prime}, x_{i} w_{i} x_{i}^{-1}=w_{i+1}$ and either $l\left(x_{i} w_{i}\right)=l\left(x_{i}\right)+l\left(w_{i}\right)$ or $l\left(w_{i} x_{i}^{-1}\right)=l\left(w_{i}\right)+l\left(x_{i}^{-1}\right)$ for all $1 \leq i \leq n-1$. We may suppose without loss of generality that $n=1$ and that there exists an $x \in W_{J}$ such that $x w x^{-1}=w^{\prime}$ and $l(x w)=l(x)+l(w)$. Note that this also implies $l\left(w^{\prime} x\right)=l\left(w^{\prime}\right)+l(x)$, since $x w=w^{\prime} x$ and $l(w)=l\left(w^{\prime}\right)$.

It follows that $\tilde{T}_{x} \tilde{T}_{w} \tilde{T}_{x}^{-1}=\tilde{T}_{w^{\prime}}$, since $\tilde{T}_{x} \tilde{T}_{w}=\tilde{T}_{x w}=\tilde{T}_{w^{\prime} x}=\tilde{T}_{w^{\prime}} \tilde{T}_{x}$. Thus

$$
\mathfrak{h}\left(\tilde{T}_{w}\right)=\mathfrak{h}\left(\tilde{T}_{x} \tilde{T}_{w} \tilde{T}_{x}^{-1}\right)=\mathfrak{h}\left(\tilde{T}_{w^{\prime}}\right)
$$

with the first equality by (3.5), and the lemma follows.

By (3.3), there exists a set of linearly independent elements of size $\left|c c l_{J}(W)\right|$ in the centralizer of $\mathcal{H}_{J}$ in $\mathcal{H}$. By (3.6), if $J$ satisfies Property II then the coefficients of the shortest elements of a $J$-conjugacy class are the same in any centralizer element, so we have as a corollary:

(3.7) Corollary. Suppose $J$ satisfies Property II. Then for any $h_{i} \in Z_{\mathcal{H}_{F}}\left(\mathcal{H}_{J}\right)$ we may write

$$
h_{i}=\sum_{j=1}^{r} a_{i, j} \tilde{T}_{\mathfrak{C}_{j, \min }}+X_{i}
$$

where $X_{i} \in \mathcal{H}_{F}$ contains no shortest elements of any $J$-conjugacy class with nonzero coefficient, and $a_{i, j} \in F$.

(3.8) Lemma. Suppose J satisfies Property II. Let $\left\{h_{i}=\sum_{j=1}^{r} a_{i, j} \tilde{T}_{\mathfrak{C}_{j, \min }}+X_{i} \in\right.$ $\left.Z_{\mathcal{H}_{F}}\left(\mathcal{H}_{J}\right) \mid \mathfrak{C}_{j} \in c l_{J}(W)\right\}$ be a set of linearly independent centralizer elements in $\mathcal{H}_{F}$, where the $X_{i}$ 's do not contain any shortest elements of any $J$-conjugacy class. Then the set $\left\{h_{i}: 1 \leq i \leq r\right\}$ is linearly independent if and only if the set of vectors $\left\{\mathbf{a}_{i}=\left(a_{i, 1}, \ldots, a_{i, r}\right) \mid 1 \leq i \leq r\right\}$ is linearly independent.

Proof. Suppose that the $\mathbf{a}_{i}$ are not linearly independent, and there is some relation $\sum_{i} r_{i} \mathbf{a}_{i}=0$ for some $r_{i} \in F$. Then $\sum_{i} r_{i} a_{i, j}=0$ for all $j$, and we have

$$
\begin{aligned}
\sum_{i} r_{i} h_{i} & =\sum_{i} r_{i} \mathbf{a}_{i} \cdot\left(\tilde{T}_{\mathfrak{C}_{1, \min }}, \ldots, \tilde{T}_{\mathfrak{C}_{r, \min }}\right)+\sum_{i} r_{i} X_{i} \\
& =\sum_{i} r_{i} X_{i} .
\end{aligned}
$$

The left hand side of the equation is in the centre, so the right hand side is also. This is a contradiction by (1.3), since $\sum_{i} r_{i} X_{i}$ has no shortest elements with non-zero coefficient.

If on the other hand the $h_{i}$ are linearly dependent, we have $\sum_{i} r_{i} h_{i}=0$ for some $r_{i} \in F$, so

$$
\sum_{i} r_{i} \mathbf{a}_{i} \cdot\left(\tilde{T}_{\mathfrak{C}_{1, \min }}, \ldots, \tilde{T}_{\mathfrak{C}_{r, \min }}\right)+\sum_{i} r_{i} X_{i}=0,
$$

and again, since $X_{i}$ contains no shortest elements, we may equate coefficients of shortest elements in $\tilde{T}_{\mathfrak{C}_{j, \text { min }}}$ to give $\sum_{i} r_{i} a_{i, j}=0$ for each $j$, so $\sum_{i} r_{i} \mathbf{a}_{i}=0$ and the $\mathbf{a}_{i}$ are linearly dependent.

(3.9) Lemma. Suppose J satisfies Property II. Then there exists an element in the centralizer $Z_{\mathcal{H}_{F}}\left(\mathcal{H}_{J}\right)$ which contains shortest elements from $\mathfrak{C}$ with coefficient 1 , and no other shortest elements from any $J$-conjugacy class. 
Proof. As pointed out above (3.3), there exist $r$ linearly independent elements $\left\{h_{i} \mid\right.$ $1 \leq i \leq r\}$ in $Z_{\mathcal{H}_{F}}\left(\mathcal{H}_{J}\right)$, and we can decompose them as in (3.7). We can then write the vector equation

$$
\left(\begin{array}{c}
h_{1} \\
\vdots \\
h_{r}
\end{array}\right)=\left(\begin{array}{ccc}
a_{1,1} & \ldots & a_{1, r} \\
\vdots & & \vdots \\
a_{r, 1} & \ldots & a_{r, r}
\end{array}\right)\left(\begin{array}{c}
\tilde{T}_{\mathfrak{C}_{1, \min }} \\
\vdots \\
\tilde{T}_{\mathfrak{C}_{r, \min }}
\end{array}\right)+\left(\begin{array}{c}
X_{1} \\
\vdots \\
X_{r}
\end{array}\right),
$$

where the $a_{i, j}$ are in $F$, and the $X_{i}$ contain no shortest elements of any $J$-conjugacy class.

By Lemma (3.8), the rows of the matrix $A=\left(a_{i, j}\right)$ are linearly independent, so $A$ is invertible, and we have

$$
A^{-1}\left(\begin{array}{c}
h_{1} \\
\vdots \\
h_{r}
\end{array}\right)=\left(\begin{array}{c}
\tilde{T}_{\mathfrak{C}_{1, \min }} \\
\vdots \\
\tilde{T}_{\mathfrak{C}_{r, \min }}
\end{array}\right)+A^{-1}\left(\begin{array}{c}
X_{1} \\
\vdots \\
X_{r}
\end{array}\right) .
$$

Each entry of the vector on the left hand side is in the centralizer, and so on the right hand side we also have a vector whose entries are centralizer elements. But each of these on the right hand side is $\tilde{T}_{\mathfrak{C}_{i, \min }}$ plus a linear combination of elements $X_{j}$ of $\mathcal{H}_{F}$, none of whom contain any shortest elements of any $J$-conjugacy class.

(3.10) Theorem. Suppose J satisfies Properties I and II. Then there exists a Jclass element $\Gamma_{\mathfrak{C}} \in Z_{\mathcal{H}}\left(\mathcal{H}_{J}\right)^{+}$. Furthermore, $\Gamma_{\mathfrak{C}}$ is the unique element of $Z_{\mathcal{H}}\left(\mathcal{H}_{J}\right)^{+}$ satisfying (3.4.1) and (3.4.2).

Proof. Recall from (3.1)(i) that if $J$ satisfies Property I, then the coefficient of any element $\tilde{T}_{w}$ in a centralizer element $h$ may be written as an $R^{+}$-linear combination of the coefficients of shortest elements from $J$-conjugacy classes in $h$. From (3.9), we have the existence of an element $h_{\mathfrak{C}}=\tilde{T}_{\mathfrak{C}_{\text {min }}}+Y \in Z_{\mathcal{H}_{F}}\left(\mathcal{H}_{J}\right)$, where $Y$ contains no shortest elements of any $J$-conjugacy class. Since the only shortest elements of any $J$-conjugacy class in $h_{\mathfrak{C}}$ have coefficient $1,(3.1)$ implies every $\tilde{T}_{w}$ occurring in $h_{\mathfrak{C}}$ has coefficient in $R^{+}$, so $h_{\mathfrak{C}} \in Z_{\mathcal{H}}\left(\mathcal{H}_{J}\right)^{+}$.

Suppose $a \tilde{T}_{w} \leq h_{\mathfrak{C}}$ with $a$ a non-zero integer. That is, it has non-zero specialization. Then by $(3.1)$ (ii) we have $a \tilde{T}_{\mathfrak{C}^{\prime}} \leq h_{\mathfrak{C}}$, where $\mathfrak{C}^{\prime}$ is the $J$-conjugacy class containing $w$. So the shortest elements of $\mathfrak{C}^{\prime}$ appear in $h_{\mathfrak{C}}$, which means $\mathfrak{C}=\mathfrak{C}^{\prime}$ since $h_{\mathfrak{C}}$ contains shortest elements of only one $J$-conjugacy class, $\mathfrak{C}$. Further, the only shortest elements in $h_{\mathfrak{C}}$ have coefficient one, so $a=1$. Thus $a \tilde{T}_{w}=\tilde{T}_{w} \leq \tilde{T}_{\mathfrak{C}}$, and so the only terms with non-zero specialization in $h_{\mathfrak{C}}$ are from $\tilde{T}_{\mathfrak{C}}$, giving $h_{\mathfrak{C}}=\tilde{T}_{\mathfrak{C}}+X$, with $X$ specializing to zero.

For uniqueness, suppose there exists a $\Gamma_{\mathfrak{C}}^{\prime} \in Z_{\mathcal{H}}\left(\mathcal{H}_{J}\right)^{+}$satisfying (3.4.1) and (3.4.2). Then $\Gamma_{\mathfrak{C}}-\Gamma_{\mathfrak{C}}^{\prime}$ has no shortest elements of any $J$-conjugacy class with non-zero coefficient. This contradicts (1.3) unless $\Gamma_{\mathfrak{C}}=\Gamma_{\mathfrak{C}}^{\prime}$.

\section{The Minimal BASIS}

With the existence of $J$-class elements for $J$ satisfying properties I and II, we now have a way, using the results of [F1, to prove the minimal basis theorem without using characters for $Z_{\mathcal{H}}\left(\mathcal{H}_{J}\right)$ for any $J$ satisfying properties I and II, via a mild generalization of the algorithm $\mathfrak{A}$ defined in [F1]. In this section, we remove the need for the isomorphism between $\mathcal{H}_{F}$ and $F W$. 
First, we recall the definition of $\mathfrak{A}$, modifying it only to the extent of including the possibility of $J \subset S$.

Suppose $h=h_{s}+h_{s}^{\prime} \in \mathcal{H}^{+}$, with $h_{s}$ a maximal element of $Z_{\mathcal{H}}\left(\tilde{T}_{s}\right)$ less than $h$. Let $m_{s}$ be the length of the shortest term in $h_{s}^{\prime}$ (for non-zero $h_{s}^{\prime}$ ).

(4.1) Definition. Let $h \in \mathcal{H}^{+}$. Define the algorithm $\mathfrak{A}_{J}$ to conduct the following procedures, for $J \subseteq S$ :

(i) split $h$ into $h=h_{s}+h_{s}^{\prime}$ for each $s \in J$ such that $h_{s}$ is maximal in $Z_{\mathcal{H}}\left(\tilde{T}_{s}\right)$ less than or equal to $h$;

(ii) if $h_{s}^{\prime}=0$ for all $s \in J$, stop;

$(\text { ii })^{\prime}$ otherwise evaluate $m_{s}$ for each $s$ such that $h_{s}^{\prime} \neq 0$, and choose $s \in J$ such that $m_{s}$ is minimal;

(iii) add terms to $h$ which complete the $s$-class elements of those terms in $h_{s}^{\prime}$ of length $m_{s}$;

(iv) declare the new element to be $\mathfrak{A}_{J}(h)$, and repeat from (i) with this new element.

The main result of [F1] relating to this algorithm is that if we start with the sum of shortest elements in a conjugacy class $\tilde{T}_{C_{\min }}$ (i.e. $J=S$ ), then at each step the elements of length $m_{s}$ in $h_{s}^{\prime}$ are all shortest in their $s$-conjugacy class, so that the $s$-class element containing them is uniquely determined, and all additions are of length $m_{s}$ or longer. This is shown to imply that there is a finite integer $n \in \mathbb{N}$ such that $\mathfrak{A}_{S}^{n}\left(\tilde{T}_{C_{\text {min }}}\right)=\Gamma_{C}$. Exactly the same argument as appears in F1] will work to prove the result for $J \subset S$, as the only facts needed for the proof are the existence of elements of form $\tilde{T}_{C}+\xi X \in Z(\mathcal{H})^{+}$, with $X$ containing no shortest elements from any conjugacy class, and the reducibility of conjugacy classes. Given analogous properties for $J \subset S$, we then have

(4.2) Theorem. Suppose $J \subseteq S$ is such that $J$ satisfies properties $I$ and II. Then the algorithm $\mathfrak{A}_{J}$ adds only same-length or longer elements when started on $\tilde{T}_{\mathfrak{C}}$, and there exists an $n \in \mathbb{N}$ such that $\mathfrak{A}_{J}^{n}\left(\tilde{T}_{\mathfrak{C}_{\min }}\right)=\Gamma_{\mathfrak{C}}$.

Use of the algorithm has several important consequences, one of which is the following:

(4.3) Lemma. If $w \in \mathfrak{C}_{\min }$ and $r \tilde{T}_{w} \leq h \in Z_{\mathcal{H}}\left(\mathcal{H}_{J}\right)^{+}$, then $r \Gamma_{\mathfrak{C}} \leq h$.

Proof. By (3.6) we have $r \tilde{T}_{\mathfrak{C}_{\text {min }}} \leq h$. The algorithm $\mathfrak{A}_{J}$ adds only same-length or longer elements when started on $\tilde{T}_{\mathfrak{C}_{\text {min }}}$, by $(4.2)$, and by [F1, 3.2] all such additions are implications inside a centralizer element. So, the same series of additions we would make if building $r \Gamma_{C}$ from $r \tilde{T}_{C_{\min }}$ becomes a series of implications, giving us $r \Gamma_{C} \leq h$.

(4.4) Proposition. If $(W, S)$ is a finite Coxeter system, and $J \subseteq S$ satisfies properties $I$ and $I I$, then the set of J-class elements is the set of primitive minimal positive elements of $Z_{\mathcal{H}}\left(\mathcal{H}_{J}\right)$. That is,

$$
Z_{\mathcal{H}}\left(\mathcal{H}_{J}\right)_{\text {min }}^{+}=\left\{\Gamma_{\mathfrak{C}} \mid \mathfrak{C} \in \operatorname{ccl}_{J}(W)\right\} .
$$

Proof. We will simply show that for any non-zero $h \in Z_{\mathcal{H}}\left(\mathcal{H}_{J}\right)^{+}$, there exists a $J$-class element $\Gamma_{\mathfrak{C}}$ such that $r \Gamma_{\mathfrak{C}} \leq h$ for some $r \in R^{+}$.

Since $h$ is non-zero, by (1.3) there is a shortest element $w$ of some $J$-conjugacy class $\mathfrak{C}$ such that $\tilde{T}_{w}$ has non-zero coefficient $r \in R^{+}$in $h$. Then by (4.3) we must have $r \Gamma_{\mathfrak{C}} \leq h$. This completes the proof. 
(4.5) Theorem. Suppose $J \subseteq S$ satisfies properties $I$ and II. Then $Z_{\mathcal{H}}\left(\mathcal{H}_{J}\right)_{\min }^{+}$is an $R$-basis for $Z_{\mathcal{H}}\left(\mathcal{H}_{J}\right)$.

Proof. The linear independence of the elements in $Z_{\mathcal{H}}\left(\mathcal{H}_{J}\right)_{\text {min }}^{+}$can be seen by specializing to $\xi_{s}=0$ for all $s \in S$. The result is a set of $J$-conjugacy class sums, a linearly independent set in the group algebra $R_{0} W$.

It remains to show spanning. We begin by showing that $Z_{\mathcal{H}}\left(\mathcal{H}_{J}\right)^{+}$is spanned by the set $Z_{\mathcal{H}}\left(\mathcal{H}_{J}\right)_{\min }^{+}$over $R^{+}$.

Let $h \in Z_{\mathcal{H}}\left(\mathcal{H}_{J}\right)^{+}$. If $h \in Z_{\mathcal{H}}\left(\mathcal{H}_{J}\right)_{\text {min }}^{+}$, then we are done, so suppose otherwise. Then $h$ is either not minimal, or is minimal with a factor of $\mathfrak{m}_{i}$ for some monomial $\mathfrak{m}_{i}$. If the latter, then we are done, as $h$ is an $R^{+}$-multiple of an element of $Z_{\mathcal{H}}\left(\mathcal{H}_{J}\right)_{\text {min }}^{+}$. If the former, then there exists a minimal element $a_{1} \in\left(Z_{\mathcal{H}}\left(\mathcal{H}_{J}\right)^{+}, \leq\right)$ such that $0 \neq a_{1}<h$. Let $k \in \mathbb{N}$ be maximal such that $\mathfrak{m}_{k}$ is a factor of $a_{1}$. Then we can write $a_{1}=\mathfrak{m}_{k} a_{1}^{\prime}$, with $a_{1}^{\prime} \in Z_{\mathcal{H}}\left(\mathcal{H}_{J}\right)_{\text {min }}^{+}$.

Then $h=h_{1}+\mathfrak{m}_{k} a_{1}^{\prime}$, where $h_{1} \in Z_{\mathcal{H}}\left(\mathcal{H}_{J}\right)^{+}$, and $h_{1}<h$.

We can now repeat the process for $h_{1}$, removing an $R^{+}$multiple of an element of $Z_{\mathcal{H}}\left(\mathcal{H}_{J}\right)_{\text {min }}^{+}$and staying in $Z_{\mathcal{H}}\left(\mathcal{H}_{J}\right)^{+}$. Thus we may continually reduce $h$ by nontrivial multiples of elements of $Z_{\mathcal{H}}\left(\mathcal{H}_{J}\right)_{\text {min }}^{+}$. This sequence of reductions will finish in a finite number of steps, as $h \in \sum_{w \in W}\left[\bigoplus_{j \in \mathbb{N}, \mathfrak{m}_{j} \in \operatorname{Mon}_{j}} \mathbb{N m}_{j}\right] \tilde{T}_{w}$, giving us $h$ as an $R^{+}$-linear combination of elements of $Z_{\mathcal{H}}\left(\mathcal{H}_{J}\right)_{\text {min }}^{+}$.

Now suppose $h \in Z_{\mathcal{H}}\left(\mathcal{H}_{J}\right)$ (not necessarily positive). Then $h=h^{+}+h^{-}$, where $h^{+} \in \mathcal{H}^{+}$, and $-h^{-} \in \mathcal{H}^{+}$. Choose $w \in W$ such that $l(w)$ is minimal for the terms in $h^{-}$. Then $w \in \mathfrak{C}$ for some $J$-conjugacy class $\mathfrak{C}$ of $W$. If $\tilde{T}_{w}$ has coefficient $-r$ in $h^{-}$for some $r \in R^{+}$, then so does $\tilde{T}_{\mathfrak{C}_{w, \text { min }}}$, by (3.6), because they have the same coefficient in $h$. If we then add $r \Gamma_{\mathfrak{C}}$ to $h$, then $-r \tilde{T}_{w}$ is no longer a term of $h+r \Gamma_{\mathfrak{C}}$. We may proceed in this way to remove all negative terms in $h$, by adding an $R^{+}$-linear combination of the $\Gamma_{\mathfrak{C}}$, giving us

$$
h+\sum_{\mathfrak{C}} r_{\mathfrak{C}} \Gamma_{\mathfrak{C}} \in Z_{\mathcal{H}}\left(\mathcal{H}_{J}\right)^{+}
$$

for some coefficients $r_{\mathfrak{C}}$ in $R^{+}$.

Then, by our above work, we may write $h+\sum_{\mathfrak{C}} r_{\mathfrak{C}} \Gamma_{\mathfrak{C}}$ as an $R^{+}$-linear combination of the $\Gamma_{\mathfrak{C}}$ also, so that $h+\sum_{\mathfrak{C}} r_{\mathfrak{C}} \Gamma_{\mathfrak{C}}=\sum_{\mathfrak{C}} r_{\mathfrak{C}}^{\prime} \Gamma_{\mathfrak{C}}$, giving us

$$
h=\sum_{\mathfrak{C}}\left(r_{\mathfrak{C}}^{\prime}-r_{\mathfrak{C}}\right) \Gamma_{\mathfrak{C}},
$$

so that $h$ is in the $R$-span of the $J$-class elements.

Finally, we are able to draw as a corollary the minimal basis theory for the centralizers of parabolic subalgebras in types $A$ and $B$, combining the results of Section 2 with those of Sections 3 and 4.

(4.6) Theorem. The set $Z_{\mathcal{H}}\left(\mathcal{H}_{J}\right)_{\text {min }}^{+}$is an R-basis for $Z_{\mathcal{H}}\left(\mathcal{H}_{J}\right)$ if (a) $\mathcal{H}$ is any type of finite Coxeter group, and $J=S$, or (b) $\mathcal{H}$ is of type $A$ or $B$ and $J \subset S$.

Further, in both the above cases the elements $\Gamma_{\mathfrak{C}}$ of $Z_{\mathcal{H}}\left(\mathcal{H}_{J}\right)_{\text {min }}^{+}$are characterized by the properties

(i) $\left.\Gamma_{\mathfrak{C}}\right|_{\xi_{s}=0, s \in S}=\tilde{T}_{\mathfrak{C}}$, and

(ii) $\Gamma_{\mathfrak{C}}-\tilde{T}_{\mathfrak{C}}$ has no shortest elements of any $J$-conjugacy class. 
Proof. This is simply a combination of theorem (1.2) (the Geck-Pfeiffer result) and theorems (2.5) and (2.6) (proving the analogy for $J$-conjugacy classes in types $A$ and $B)$, and (3.10) and (4.5).

\section{REFERENCES}

[C] R. Carter, Representation theory of the 0-Hecke algebra, J. Algebra 104 (1986), 89-103. MR 88a:20014

[DD] R. Dipper and J. Du, Trivial and alternating source modules of Hecke algebras of type A, Proc. London Math. Soc. 66 (3) (1993), 479-506. MR 94a:20022

[F1] A. Francis, The minimal basis for the centre of an Iwahori-Hecke algebra, J. Algebra 221 (1999), 1-28. MR 2000k:20005

[F2] Centralizers of Iwahori-Hecke algebras II: the general case, preprint.

[GHLMP] M. Geck, G. Hiss, F. Lübeck, G. Malle, and G. Pfeiffer, CHEVIE-a system for computing and processing generic character tables. Computational methods in Lie theory (Essen 1994), Appl. Algebra Engrg. Comm. Comput. 7 (3) (1996), 175-210. MR 99m:20017

[GP] M. Geck and G. Pfeiffer, On the irreducible characters of Iwahori-Hecke algebras, Adv. Math. 102 (1993), 79-94. MR 94m:20018

[GR] M. Geck and R. Rouquier, Centers and simple modules for Iwahori-Hecke algebras, in Finite Reductive Groups (Luminy, 1994), Prog. Math., vol. 141, Birkhäuser, Boston, MA, 1997, pp. 251-272. MR 98c:20013

[J] L. Jones, Centers of generic Hecke algebras, Trans. Amer. Math. Soc. 317 (1990), 361-392. MR 90d:20030

[R] A. Ram, A Frobenius formula for the characters of the Hecke algebras, Invent. Math 106 (1991), 461-488. MR 93c:20029

Department of Mathematics, University of Virginia, Charlottesville, Virginia 22903

Current address: University of Western Sydney, Richmond, NSW 2753, Australia

E-mail address: a.francis@uws.edu.au 